Open Access

\title{
Microbiomes of the dust particles collected from the International Space Station and Spacecraft Assembly Facilities
}

Aleksandra Checinska', Alexander J. Probst², Parag Vaishampayan', James R. White ${ }^{3}$, Deepika Kumar ${ }^{4}$, Victor G. Stepanov ${ }^{4}$, George E. Fox ${ }^{4}$, Henrik R. Nilsson ${ }^{5}$, Duane L. Pierson ${ }^{6}$, Jay Perry ${ }^{7}$ and Kasthuri Venkateswaran ${ }^{1 *}$

\begin{abstract}
Background: The International Space Station (ISS) is a unique built environment due to the effects of microgravity, space radiation, elevated carbon dioxide levels, and especially continuous human habitation. Understanding the composition of the ISS microbial community will facilitate further development of safety and maintenance practices. The primary goal of this study was to characterize the viable microbiome of the ISS-built environment. A second objective was to determine if the built environments of Earth-based cleanrooms associated with space exploration are an appropriate model of the ISS environment.
\end{abstract}

Results: Samples collected from the ISS and two cleanrooms at the Jet Propulsion Laboratory (JPL, Pasadena, CA) were analyzed by traditional cultivation, adenosine triphosphate (ATP), and propidium monoazide-quantitative polymerase chain reaction (PMA-qPCR) assays to estimate viable microbial populations. The 165 rRNA gene Illumina iTag sequencing was used to elucidate microbial diversity and explore differences between ISS and cleanroom microbiomes. Statistical analyses showed that members of the phyla Actinobacteria, Firmicutes, and Proteobacteria were dominant in the samples examined but varied in abundance. Actinobacteria were predominant in the ISS samples whereas Proteobacteria, least abundant in the ISS, dominated in the cleanroom samples. The viable bacterial populations seen by PMA treatment were greatly decreased. However, the treatment did not appear to have an effect on the bacterial composition (diversity) associated with each sampling site.

Conclusions: The results of this study provide strong evidence that specific human skin-associated microorganisms make a substantial contribution to the ISS microbiome, which is not the case in Earth-based cleanrooms. For example, Corynebacterium and Propionibacterium (Actinobacteria) but not Staphylococcus (Firmicutes) species are dominant on the ISS in terms of viable and total bacterial community composition. The results obtained will facilitate future studies to determine how stable the ISS environment is over time. The present results also demonstrate the value of measuring viable cell diversity and population size at any sampling site. This information can be used to identify sites that can be targeted for more stringent cleaning. Finally, the results will allow comparisons with other built sites and facilitate future improvements on the ISS that will ensure astronaut health.

Keywords: International Space Station, Air, Surface, Microbiome, Closed habitat, Cleanroom, Propidium monoazide

\footnotetext{
* Correspondence: kjvenkat@jpl.nasa.gov

1 Jet Propulsion Laboratory, Biotechnology and Planetary Protection Group, California Institute of Technology, M/S 89-2 4800 Oak Grove Dr., Pasadena, CA 91109, USA

Full list of author information is available at the end of the article
}

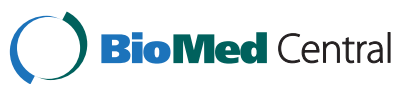

(C) 2015 Checinska et al. Open Access This article is distributed under the terms of the Creative Commons Attribution 4.0 International License (http://creativecommons.org/licenses/by/4.0/), which permits unrestricted use, distribution, and reproduction in any medium, provided you give appropriate credit to the original author(s) and the source, provide a link to the Creative Commons license, and indicate if changes were made. The Creative Commons Public Domain Dedication waiver (http://creativecommons.org/publicdomain/zero/1.0/) applies to the data made available in this article, unless otherwise stated. 


\section{Background}

The microbial characterization of the International Space Station (ISS) has been mostly limited to traditional culture-based microbiology and selective molecular biology methods, such as Sanger sequencing, for supporting tasks such as water remediation, food safety, and crewmember health [1-5]. Since built environments are known to have specific microbiomes [6], it is of the highest interest to the National Aeronautics and Space Administration (NASA) scientific community to explore the environmental microbiome of the ISS as a closed environment. Moreover, the National Research Council (NRC) specifically recommended that NASA study changes in microbial populations in response to selective pressure associated with microgravity, which characterizes life aboard the ISS [7]. Previous studies show that permanent changes have occurred within the microbial species during experiments aboard the ISS $[8,9]$. Although next-generation sequencing (NGS) analyses are now broadly implemented in many microbiology-related scientific fields, especially in microbial ecology [10, 11] and human microbiome projects $[12,13]$, use of these techniques for closed habitats has just begun [14] and warrants more research.

"Deep" sequencing of ISS samples can answer questions on abundance and diversity of the microorganisms. However, differentiating viable and yet-to-be-cultivable microbial populations requires an appropriate sample processing technology [15]. The use of the reagent propidium monoazide (PMA) before DNA extraction eliminates cells with a compromised membrane. The PMA-based assay thus allows for a more accurate approximation of the viable microbial community in terms of richness as well as abundance [15]. Due to the technically rigorous methods required for culturing many microorganisms, characterization of human-associated microbial populations in the ISS environment remains a significant challenge. However, it is important to monitor the presence of any opportunistic pathogenic microorganisms. As longduration human missions are planned in the future, detection of human pathogens and possible mitigation practices must be developed. In addition, understanding of the ISS microbiome could facilitate the necessary maintenance of this closed habitat and thereby assist in preventing degradation of its components by some microorganisms [4].

Both ISS environment and cleanrooms on Earth are maintained by high-efficiency particulate arrestance (HEPA) filters. Importantly, considerable prior knowledge of the microbial communities associated with these cleanrooms exists from prior studies. The ISS has been exposed to these communities indirectly by the fact that some of the cargos sent to the ISS were packaged in these specific NASA cleanrooms. As in the case of the ISS closed habitat, the Earth-based cleanrooms are controlled for humidity, temperature, and circulation. Furthermore, NASA quality assurance engineers perform periodic audits to ensure that certified-facility cleanliness levels conform to the requirements delineated for both the ISS and Earth cleanrooms. The ISS environment is zero gravity with exposure to space radiation and elevated carbon dioxide levels. By necessity the air is recirculated whereas on Earth the cleanrooms are constantly replenished with fresh air. An important difference is human habitation, which does not occur in the Earth cleanrooms. However, the human traffic in Earth cleanrooms is actually rather high (at least $50+$ people in a given working day) when compared to the ISS where only 6 astronauts are allowed at a single time.

This study is the first to analyze samples from the ISS air and surface using the traditional (colony counts [i.e., cultivable bacteria]) and state-of-the-art molecular techniques (adenosine triphosphate [ATP] and quantitative polymerase chain reaction [qPCR] assays) to measure the abundance of microorganisms (i.e., live and dead cells). Furthermore, the abundance and diversity of viable bacterial community were assessed using molecular methods (PMA treatment followed by qPCR [i.e., bacterial burden] or Illumina-based 16S rRNA sequencing [i.e., microbial diversity]). Additionally, the microbial diversity of the ISS was compared with samples from Jet Propulsion Laboratory (JPL) spacecraft assembly facility (SAF) cleanrooms, which also represent closed and environmentally controlled built ecosystems.

\section{Results}

\section{Microbial burden}

The samples analyzed during this study were the following: ISS HEPA filter particulates, vacuum cleaner bag components of ISS (ISS Debris), JPL Class $10 \mathrm{~K}$ cleanroom (JPL-SAF Debris), and JPL Class $1 \mathrm{~K}$ cleanroom (JPL-103 Debris) (Table 1). Microbial burdens estimated via traditional cultivation-dependent and cultivationindependent methods are given in Table 2. The bacteria capable of growth in nutrient-rich media were in the range of $10^{5} \mathrm{CFU}$ per gram in the ISS HEPA filter sample and an order of magnitude greater $\left(10^{6} \mathrm{CFU}\right.$ per gram) for the ISS Debris. Relatively, the bacterial populations were larger in the ISS Debris sample than in the JPL cleanroom debris samples. When compared to the ISS Debris, the JPL-SAF Debris harbored an order of magnitude smaller bacterial population $\left(10^{5} \mathrm{CFU}\right.$ per gram) and the JPL-103 Debris sample was two orders of magnitude fewer $\left(1.4 \times 10^{4} \mathrm{CFU}\right.$ per gram). In general, cultivable fungi were less represented than bacteria in all samples tested. The cultivable fungal population was two logs less for the ISS samples when compared to their bacterial counts, whereas the JPL cleanroom samples exhibited one-log difference. The ISS Debris 
Table 1 Characteristics of samples collected from ISS cabin locations and Earth-based cleanrooms where spacecraft components are assembled

\begin{tabular}{|c|c|c|c|c|c|c|c|c|c|}
\hline Sample name & Replicates & Location & Cleanroom classification & Source & Air/surface & Specifications & Usage time & Model & Mission activities \\
\hline ISS HEPA & 1 & ISS & None & Filter element particles & Air & $\begin{array}{l}\text { HEPA rated, retains } \\
99.97 \% \text { particles } 0.3 \mu \mathrm{m} \text {; } \\
20-\text { mesh inlet screen has } \\
841 \mu \mathrm{m} \text { sieve openings }\end{array}$ & 40 months & $\begin{array}{l}\text { Part no. SV810010-1, } \\
\text { Serial no. 0049; HEPA } \\
\text { media supplied by } \\
\text { Flanders Filters, Inc.; } \\
\text { Nomex inlet screen }\end{array}$ & $\begin{array}{l}\text { Returned aboard } \\
\text { STS-134/ULF6 in } \\
\text { May 2011 }\end{array}$ \\
\hline ISS Debris & 2 & ISS & None & $\begin{array}{l}\text { vacuum cleaner } \\
\text { bag dust }\end{array}$ & Surface & $\begin{array}{l}\text { Vacuum bag retains } \\
\text { particles }>6 \mu \mathrm{m} ; \\
\text { HEPA-rated filter } \\
\text { retains particles } \\
>0.3 \mu \mathrm{m}\end{array}$ & 1 day & ISS vacuum cleaner & $\begin{array}{l}\text { Expedition 31; } \\
\text { returned aboard } \\
\text { Soyuz flight } 295 \\
\text { in July } 2012\end{array}$ \\
\hline JPL-SAF Debris & 2 & JPL-SAF & $10 \mathrm{~K}$ & Vacuum bag dust & Surface & $\begin{array}{l}\text { Retains } 99.7 \% \\
\text { particles }>3 \mu \mathrm{m}\end{array}$ & 70 days & Nilfisk GM80, 81620000 & No major mission \\
\hline JPL-103 Debris & 1 & JPL-103 & $1 \mathrm{~K}$ & Vacuum bag dust & Surface & $\begin{array}{l}\text { Retains } 99.7 \% \\
\text { particles }>3 \mu \mathrm{m}\end{array}$ & $>180$ days & Nilfisk GM80, 81620000 & No major mission \\
\hline
\end{tabular}

Table 2 Total and viable microbiological characteristics of particles accumulated in ISS and other Earth-based cleanrooms

\begin{tabular}{|c|c|c|c|c|c|c|c|c|}
\hline \multirow[t]{2}{*}{ Sample } & \multicolumn{2}{|c|}{$\begin{array}{l}\text { Cutivable bacterial population } \\
\text { (CFU/g) that are: }\end{array}$} & \multicolumn{2}{|c|}{$\begin{array}{l}\text { qPCR-based bacterial population } \\
\text { (16S rRNA copies/g) }\end{array}$} & \multirow[t]{2}{*}{$\begin{array}{l}\text { Viable bacterial population } \\
(\mathrm{B} / \mathrm{A} \times 100)\end{array}$} & \multicolumn{2}{|c|}{$\begin{array}{l}\text { ATP-based microbial population } \\
\text { (RLU/g): }\end{array}$} & \multirow[t]{2}{*}{$\begin{array}{l}\text { Viable microbial population } \\
(\mathrm{D} / \mathrm{C} \times 100)\end{array}$} \\
\hline & Bacteria & Fungi & Untreated (A) & PMA-treated (B) & & Total ATP (C) & Intracellular ATP (D) & \\
\hline ISS HEPA & $8.17 \times 10^{5}$ & $2.34 \times 10^{3}$ & $1.7 \times 10^{9}$ & $2.91 \times 10^{7}$ & 1.7 & $2.06 \times 10^{7}$ & $3.54 \times 10^{5}$ & 1.7 \\
\hline ISS Debris & $1.34 \times 10^{6}$ & $5.02 \times 10^{4}$ & $4.5 \times 10^{8}$ & $1.21 \times 10^{7}$ & 2.7 & $3.50 \times 10^{7}$ & $2.65 \times 10^{7}$ & 75.7 \\
\hline JPL-SAF Debris & $1.28 \times 10^{5}$ & $5.05 \times 10^{4}$ & $3.1 \times 10^{8}$ & $2.07 \times 10^{8}$ & 66.8 & $1.91 \times 10^{7}$ & $2.10 \times 10^{6}$ & 11.0 \\
\hline JPL-103 Debris & $1.40 \times 10^{4}$ & $3.30 \times 10^{3}$ & $4.3 \times 10^{8}$ & $1.90 \times 10^{7}$ & 4.5 & $2.60 \times 10^{6}$ & $1.20 \times 10^{6}$ & 46.2 \\
\hline
\end{tabular}


possessed a similar fungal burden as that of the JPL-SAF Debris sample, which is higher than the more stringent JPL-103 area. The ISS HEPA harbored less fungi when compared to the ISS Debris.

The qPCR-based assay, which measured DNA from both dead and live cells, estimated that the ISS HEPA sample had the highest bacterial density $\left(1.7 \times 10^{9} 16 \mathrm{~S}\right.$ rRNA copies per grams) compared to all other samples, which were at least one-log less abundant in total bacterial burden. This trend was not noticed in the samples that were treated with PMA (viable bacterial population), where $\sim 10^{7} 16 \mathrm{~S}$ rRNA gene copies per gram accounted for all samples except in the JPL-SAF, which exhibited $\sim 10^{8} 16 \mathrm{~S}$ rRNA gene copies. The percentage of the viable bacterial population (PMA-treated samples) was $\sim 1.7 \%$ in the ISS HEPA, $\sim 2.7 \%$ in ISS Debris, and $\sim 4.5 \%$ in the JPL-103 samples. However, approximately $67 \%$ of the bacteria in the JPL-SAF Debris were viable.

The total ATP contents derived from both dead and alive cells were in the range of $10^{7}$ relative light unit (RLU) per gram, except for the sample collected from the Class $1 \mathrm{~K} \mathrm{JPL}-103$ cleanroom, which showed $\sim 2.6 \times 10^{6}$ RLU per gram. The microbial estimation carried out via an intracellular ATP assay (viable cells only) indicated that only $1.7 \%$ of the total microorganisms were viable in the ISS HEPA sample. In the case of the ISS Debris sample, this percentage was much higher (75\%); similarly, JPL-SAF and JPL-103 constituted approximately $11 \%$ and $46 \%$ viable microorganisms, respectively.

\section{Culture-based microbial diversity}

The 16S rRNA sequencing-based identification and phylogenetic affiliation of the bacterial and fungal strains isolated during this study are given in Additional file 1: Table S1. Among 41 bacterial strains isolated and identified, 36 belonged to 29 different species, five strains were only identified to the genus level. The majority of the identified isolates belong to Firmicutes and only four strains were affiliated to the members of the Proteobacteria group. The highest number of isolates was represented by Bacillus and Staphylococcus genera. While Bacillus was dominant in both ISS samples, Staphylococcus was only present in ISS Debris. Both genera were either absent or underrepresented in the JPL-SAF and JPL-103 samples. Single representatives of other spore-forming lineages were also identified (Paenibacillus, Brevibacillus, and Solibacillus). Notably, five strains belonging to the B. anthracis-cereusthuringiensis group were isolated from the HEPA filter, and more detailed phylogenetic, pathogenic, and whole genome sequence characterizations are underway to confirm the functional characteristics of these isolates. The pathogenic properties specific to $B$. anthracis were not present in these five strains (data not shown).
Among 19 fungal strains sequenced, 13 strains were identified to six different species. Out of 19 fungal strains, 16 were isolated from the ISS samples and the other three strains were from JPL samples. All the cultivated fungal strains affiliated phylogenetically to the members of the Ascomycota phylum. The most common fungal isolate in the ISS samples was Aspergillus niger, although other diverse species of Aspergillus were also identified, along with Penicillium as the second most dominant genus (Additional file 1: Table S1). Based on these results, most of the cultivated bacteria or fungi from the ISS locations were not noticed in the Earthanalog cleanrooms.

\section{Pyrosequencing-derived bacterial diversity}

The number of pyrosequences belonging to various bacterial phyla and operational taxonomic units (OTUs) is presented in Additional file 1: Table S2. Approximately, 100,000 reads of bacterial pyrosequences of $>500 \mathrm{bp}$ in length were generated from eight samples during this study. When the software mothur [16] was employed for the bioinformatics analysis, $\sim 71 \%$ of the sequences were deemed to be of good quality and used for further analysis, resulting in 70,669 sequences (Additional file 1: Table S2).

Among PMA-untreated samples, the ISS Debris yielded the highest number of sequences (30,111 reads) whereas the lowest number of sequences was seen in the ISS HEPA $(1,720)$. Likewise, more sequences were retrieved from the JPL-SAF (8,482 reads) as compared with the JPL-103 $(5,062)$ sample. Contrary to the pyrosequence abundance, higher numbers of OTUs were present in the JPL-SAF sample (1,448 OTUs) than in the ISS Debris (452 OTUs) sample. However, as noticed in the pyrosequence abundance, the number of OTUs was also higher in the JPL-103 sample (301 OTUs) as compared to the ISS HEPA (133 OTUs) sample.

The PMA-untreated samples constituted $\sim 65 \%$ of the high-quality sequences, while only $35 \%$ of the sequences were retrieved from PMA-treated samples (25,294 sequences). Among the PMA-treated (viable) portions, ISS HEPA and ISS Debris samples possessed $\sim 38 \%$ and $60 \%$ of sequences, respectively. Similar to the ISS Debris sample, a higher percent of viable sequences was retrieved from the JPL-SAF Debris (77 \%) but surprisingly, only $\sim 3 \%$ of the viable sequences were from the PMA-treated JPL-103 Debris sample.

Almost all of the viable bacterial pyrosequences retrieved from the ISS HEPA filter were members of Corynebacterium. This organism was also dominant in the ISS Debris sample (Additional file 1: Table S2). Genera such as Propionibacterium and Staphylococcus were only present in the ISS Debris sample. While the ISS-HEPA sample contained fewer sequence reads representing only 
five genera, the ISS Debris sample was dominated by low abundance of more diverse genera (Stenotrophomonas, Sphingomonas, Pseudomonas, Delftia). Bacillus species were predominant when traditional cultivation methods were employed (Additional file 1: Table S1), but Bacillus pyrosequences were not retrieved. When PMA-treated samples from the ISS samples were compared to the Earth-analog cleanroom samples, the dominant phyla were found to be very similar (Actinobacteria, Proteobacteria, Firmicutes). However, on the genus level, the JPL-SAF yielded many more viable sequences representing diverse genera (data not shown).

\section{Illumina-derived bacterial diversity}

The Illumina sequencing generated $\sim 6.8$ million highquality reads, which is $\sim 100$ times more coverage than was obtained with pyrosequencing yield Additional file 2: Figure S1. Since Illumina sequences were short ( 150 bp each), it was not possible to reliably resolve taxonomic affiliation beyond the genus level. We characterized highquality reads using the Ribosomal Database Project (RDP) classifier [17] and summarized community composition at multiple taxonomic levels. Among the ISS-associated samples, profiles were dominated by Actinobacteria, Bacilli, and Clostridia, while samples from the JPL-associated sites maintained higher levels of Alphaproteobacteria and Gammaproteobacteria (Fig. 1). At the family level, the differences between ISS and JPL profiles were substantial, with complete distinction between the two sites using actinobacterial members alone-the ISS samples were dominated by Corynebacterium, while the actinobacterial groups among JPL samples were predominantly Geodermatophilaceae.

Actinobacteria, Firmicutes, and Proteobacteria phyla together constituted $80 \%$ or more in all cases. When compared on the family level, sequences associated with any family did not exceed more than $10 \%$ of the total sequences. Characteristics of bacterial phyla that are dominant during this study are depicted in Table 3. A clear-cut trend was noticed on the presence of bacterial phylum dominance on the ISS as well as in the Earth-analog cleanroom samples. Viable sequences arising from Actinobacteria were the most abundant in the ISS HEPA and ISS Debris samples ( 95 and $\sim 66 \%$, respectively), while Proteobacteria was present in the least numbers (0.41 and $\sim 3 \%$, respectively). Among these three dominant phyla, the lowest percentage was found for Firmicutes and differed significantly between cleanroom samples as well (JPL-SAF: $11.05 \%$ and JPL-103: $0.98 \%$ ). In-depth analysis showed that the ISS HEPA sample accumulated more sequences of the genus Corynebacterium than ISS Debris sample. In addition to the latter, the ISS Debris had sequences associated with the order Actinomycetales and the genus Staphylococcus. In contrast, the JPL-SAF Debris sample possessed larger numbers of Bacillus and unclassified Proteobacteria genera sequences, whereas JPL-103 Debris mimicked ISS locations and members of Actinobacteria were more dominant.

In order to detect differentially abundant taxonomic groups between PMA-treated and untreated samples at the phylum through genus levels, several statistical analyses were conducted (see "Methods" section). Hierarchical clustering of samples using genus taxonomic profiles resulted in clustering of samples by collection site but not strong clustering of paired PMA or no PMA samples (Fig. 2). Both the negative binomial test and Fisher's exact test provided valuable information and supported the hierarchical clustering scenario. Searching for differentially abundant genus-level groups with significant $P$ values using the negative binomial test, we saw that the vast majority of differentially abundant taxa were relatively depleted in the PMA-treated group. The only well-represented genera that were relatively enriched were Clostridium ( $0.34 \%$ vs. $2.46 \%, P=0.009$ ) and Rheinheimera (0.005 \% vs. $0.472 \%, P=7 \mathrm{E}-06)$. In contrast, genera such as Streptococcus, Veillonella, Lactobacillus, Bifidobacterium, and Neisseria were all relatively enriched in the untreated samples. Alpha diversity estimators also indicated a significant drop in diversity associated with PMA treatment. On average, PMA samples had $48 \%$ fewer OTUs than their untreated pairs (paired $T$-test $P=0.004$ ). This consistent reduction in the PMA samples was also true for Faith's diversity, Chao1, and Shannon diversity measures (Fig. 3) and is consistent with the depletion of many taxa identified by differential abundance analysis.

To better characterize the shared community composition among samples, beta-diversity metrics (e.g., BrayCurtis distances) were computed and evaluated using principal coordinate analysis (PCoA). Figure 4 displays PCoA plots associated with the R1 dataset. The samples from the same site are highly similar in composition regardless of PMA treatment. Using Procrustes analysis, we compared PCoA plots generated for both the R1 and R2 datasets. In this case, Procrustes analysis was employed to transform the R2-based principal coordinate set (by rotation, scaling, and translation) to minimize the distances between corresponding points in the R1 principal coordinate space. We found that virtually identical distances were derived between the R1 and R2 PCoA plots, further supporting the clustering of PMA and untreated paired samples. This high-resolution comparison stood in contrast to broader composition comparisons, such as the hierarchical clustering above. At the OTU level, the bacterial composition was strongly associated with the sampling site, and the reduced 
overall diversity of PMA treatment did not dramatically impact the composition relative to sample origin.

\section{Pyrosequencing-derived archaeal diversity}

When the samples were subjected to the archaeal characterization, neither qPCR nor PCR for nextgeneration sequencing generated archaeal amplification products (data not shown). It is likely that the presence of archaea in these samples was at a very low concentration $(<100$ gene copies per PCR reaction), below the detection limits of the technology [14]. Alternatively, materials associated with the dust samples might have inhibited the archaeal DNA amplification. However, this is unlikely because the dust samples spiked with a purified archaeal DNA did exhibit an appropriate band after PCR amplification (data not shown).

\section{Pyrosequencing-derived fungal diversity}

In total, $\sim 35,000$ fungal pyrosequences were retrieved from the eight samples. The detailed breakdown of the number of sequences and OTUs is given in Table 4. The comparison is made on the class level, as fungal taxonomy is not as well described on the genus level as bacterial taxonomy when it comes to sequencing of environmental substrates. The fungal OTUs represented 153 distinct taxa. Most OTUs were obtained for three classes: Dothideomycetes, Eurotiomycetes, and Tremellomycetes. As noticed for the bacterial abundance, fungal species richness was also location specific. The dry cleanroom surfaces were found to harbor different fungal species (mostly Dothideomycetes and Eurotiomycetes) whereas ISS samples possessed more Eurotiomycetes, Saccharomycetes, and Exobasidiomycetes members.

When treated with PMA, no viable fungal sequences were retrieved from the ISS HEPA sample. In contrast, viable fungal sequences for Dothideomycetes and Tremellomycetes were common in the ISS Debris samples. Viable fungal diversity was far higher in the JPL-SAF Debris sample than in JPL-103 Debris. The JPL-SAF Debris sample revealed the highest diversity that are viable and the highest number of sequences. The great number of Eurotiomycetes sequences was confirmed by cultivation of Aspergillus and Penicillium isolates from the ISS HEPA and ISS debris.

\section{Significant differences of microbial communities between} ISS and Earth cleanroom samples

The differences between the ISS and Earth-analog cleanroom microbiomes were assessed using multivariate statistics. Independent analyses of bacterial sequences derived from pyrosequencing and Illumina sequencing revealed a significant difference in the community profile of ISS and Earth-analog cleanroom microbiomes. These differences are displayed in ordination analyses (Additional file 3: Figure S2) and are supported by Permutational Multivariate Analysis of Variance (PERMANOVA) and Multi-Response Permutation Procedure (MRPP) analyses (Additional file 1: Table S4). Irrespective of the sample origin, the viable community profile was highly similar to the total community profile, revealing no significant differences in PERMANOVA or MRPP indices. However, the differences between the viable and total community profile were outweighed by the substantial differences of the ISS and Earth-analog cleanroom microbiome, which ranged from 0.1 to 0.5 concerning the chance-corrected within-group agreement (Additional file 1: Table S4). Bacterial and fungal taxa that showed a significant difference between ISS and Earth-analog cleanroom samples are presented in Additional file 3: Figure S2.

\section{Discussion}

The safety and health of spaceflight crewmembers are of the highest importance for current and future missions. Individuals living and/or working in built environments are often susceptible to health issues associated with microorganisms [18, 19]. Moreover, the microbial ecology of ISS remains largely unknown, as study efforts have been mostly focused on microbiological surveillance using cultivation procedures. The NRC recommended use of state-of-the-art molecular biology techniques to develop better microbial monitoring of future closed habitat(s) and response system(s) against potential biohazards originating from microbiological sources, using the ISS as a test bed [1,4]. Exploration of the microbial diversity associated with unusual built-in environments such as the ISS would further contribute to the indoor microbiome research. This will benefit the development of spaceflight applications as well as basic and applied research on Earth.

The main goal of this study was to determine ISS air and surface viable microbiomes and unveil the differences of viable microbiomes on the ISS and Earth-based cleanrooms. The ISS is a very unique environment due to microgravity, and a suitable Earth analog of this ecosystem is not available. The cleanrooms used for comparison in this study are environmentally controlled and represent oligotrophic conditions in an Earth-based setting. Comprehensive characterization and cataloguing of microbial species in NASA cleanrooms has been taking place since the 1970s. This extensive data set renders these facilities the best-characterized closed environments with limited human traffic that the ISS samples can be compared with [20-24]. A lower incidence of cultivable microorganisms in Earth cleanrooms than ISS surfaces suggests that regular thorough maintenance 


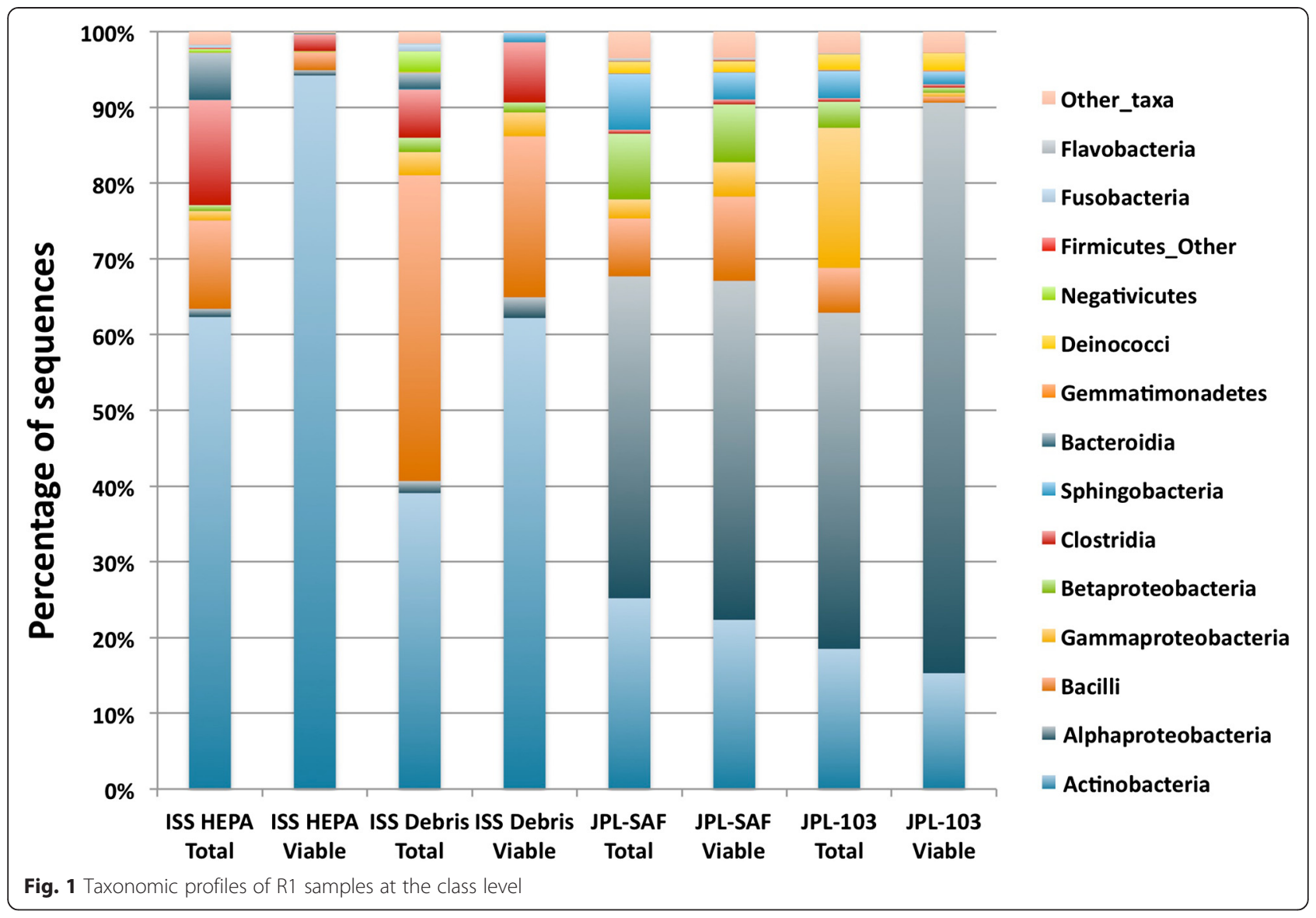

Table 3 Characteristics of dominant bacterial phyla as measured by Illumina iTag method during this study

\begin{tabular}{|c|c|c|c|c|c|c|c|c|}
\hline & $\begin{array}{l}\text { ISS HEPA } \\
\text { total }\end{array}$ & $\begin{array}{l}\text { ISS HEPA } \\
\text { viable }\end{array}$ & $\begin{array}{l}\text { ISS Debris } \\
\text { total }\end{array}$ & $\begin{array}{l}\text { ISS Debris } \\
\text { viable }\end{array}$ & $\begin{array}{l}\text { JPL-SAF } \\
\text { total }\end{array}$ & $\begin{array}{l}\text { JPL-SAF } \\
\text { viable }\end{array}$ & $\begin{array}{l}\text { JPL-103 } \\
\text { total }\end{array}$ & $\begin{array}{l}\text { JPL-103 } \\
\text { viable } \\
\end{array}$ \\
\hline Total number of sequences & 553,176 & 587,569 & $1,148,047$ & $1,116,419$ & $1,029,984$ & $1,472,777$ & 489,047 & 397,607 \\
\hline $\begin{array}{l}\text { Percentage of sequences that } \\
\text { belong to three dominant phyla }\end{array}$ & 90.92 & 99.65 & 92.35 & 98.26 & 80.25 & 81.84 & 87.96 & 90.46 \\
\hline \multicolumn{9}{|l|}{ Actinobacteria } \\
\hline Percentage of sequences & 63.28 & 95.28 & 40.52 & 66.54 & 28.76 & 25.25 & 23.79 & 21.46 \\
\hline Number of genera & 78 & 55 & 62 & 38 & 122 & 116 & 103 & 71 \\
\hline Number of dominant genera (>100 sequences) & 16 & 7 & 28 & 16 & 71 & 76 & 28 & 24 \\
\hline \multicolumn{9}{|l|}{ Firmicutes } \\
\hline Percentage of sequences & 24.83 & 3.97 & 45.67 & 28.48 & 7.70 & 11.05 & 6.60 & 0.98 \\
\hline Number of genera & 118 & 67 & 100 & 31 & 152 & 150 & 101 & 53 \\
\hline Number of dominant genera (>100 sequences) & 50 & 17 & 65 & 18 & 53 & 69 & 15 & 7 \\
\hline \multicolumn{9}{|l|}{ Proteobacteria } \\
\hline Percentage of sequences & 2.81 & 0.41 & 6.16 & 3.24 & 43.80 & 45.55 & 57.57 & 68.02 \\
\hline Number of genera & 95 & 65 & 89 & 30 & 189 & 191 & 143 & 92 \\
\hline Number of dominant genera (>100 sequences) & 22 & 7 & 49 & 10 & 96 & 104 & 56 & 29 \\
\hline
\end{tabular}




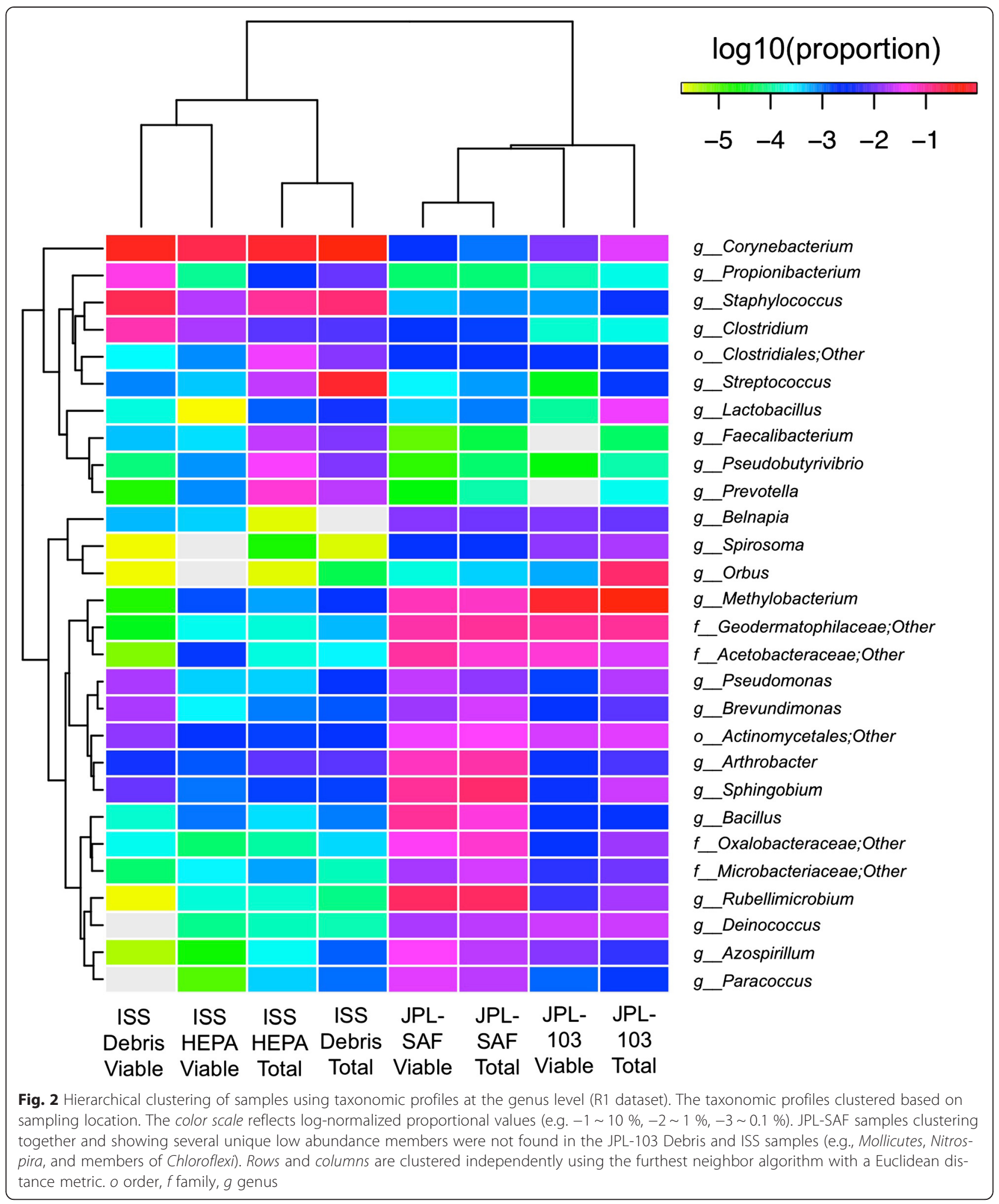

and cleaning practices are required to reduce microbial burden in closed habitats.

Multiple studies have used PMA for the estimation of viable bioburden, but they were mostly interpreted by
qPCR [25-27], next-generation sequencing [15, 28, 29], or PhyloChipG3 [15]. The utilization of PMA combined with Illumina MiSeq technology for exploring viable microbiome of ISS-related environmental surfaces is not 


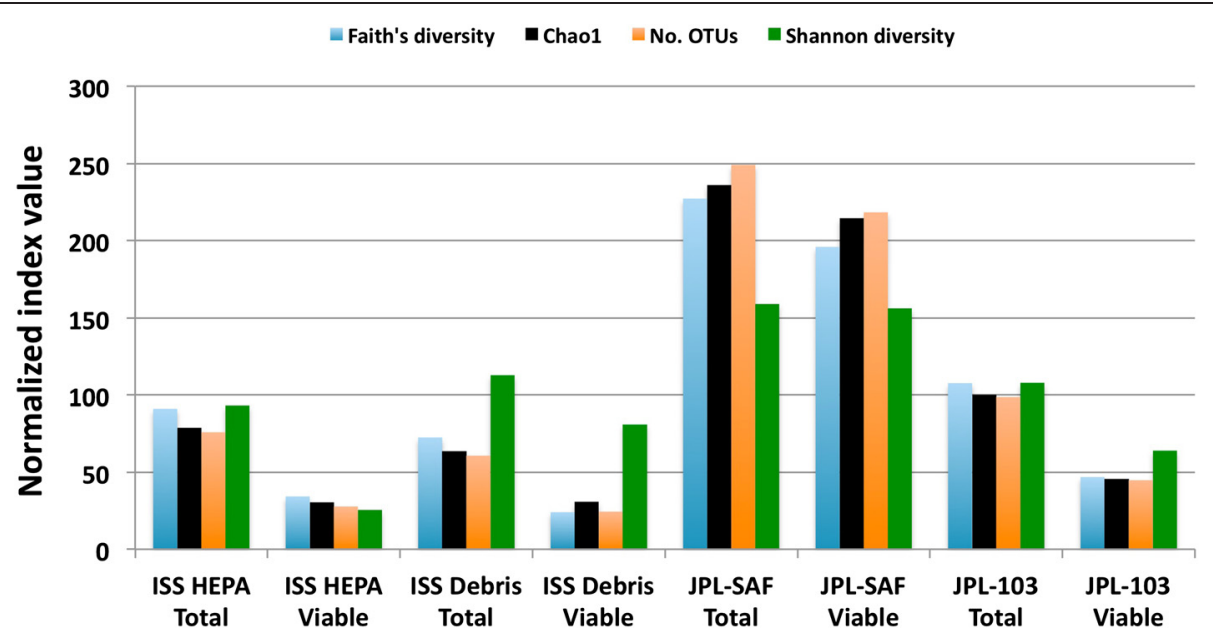

Fig. 3 PMA treatment is associated with a reduction in alpha diversity. Alpha diversity values are normalized as a percentage of their mean value across all eight samples in the chart

yet reported. The NGS technology resulting in information on millions of base pairs might have included genetic information from dead cells including contaminant DNA associated with the sample processing reagents $[30,31]$. Thus, PMA treatment would eliminate these contaminants, making it possible to elucidate viable microbiomes. However, it was documented that PMA might not work for all kinds of microbial populations, especially spores where the dye was not able to penetrate the dead but intact spores [32]. Despite this, none of the other current methodologies can precisely estimate viable populations; PMA treatment has the highest potential to lead to the substantial differentiation between dead and live cells [15]. The statistical analysis of the viable bacterial community in this study revealed the significant variance in viable microbiome (Fig. 4; Additional file 1: Table S3 and S4), between the ISS and the cleanroom samples.

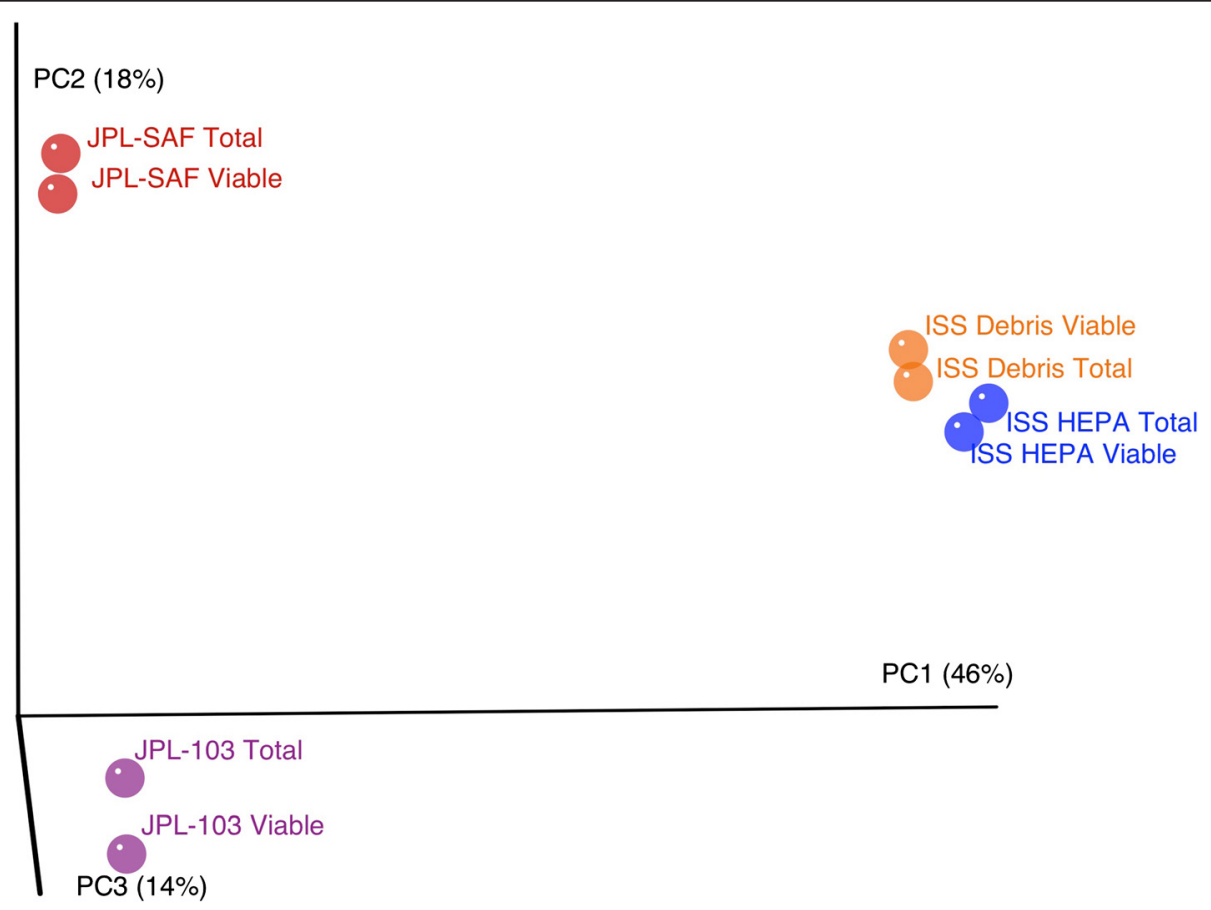

Fig. 4 Principal coordinate analysis plot (R1 data) based on Bray-Curtis distances. Percentage of variance explained by each principal coordinate axis is shown in parentheses 
Table 4 Pyrosequencing-based fungal phyla present in ISS and Earth-based cleanroom samples

\begin{tabular}{|c|c|c|c|c|c|c|c|c|c|}
\hline \multirow{2}{*}{$\begin{array}{l}\text { Phylum/class } \\
\text { (number of genera) }\end{array}$} & \multicolumn{9}{|c|}{ Number of pyrosequences that are retrieved from: } \\
\hline & $\begin{array}{l}\text { Number } \\
\text { of OTU }\end{array}$ & $\begin{array}{l}\text { ISS HEPA } \\
\text { total }\end{array}$ & $\begin{array}{l}\text { ISS HEPA } \\
\text { viable }\end{array}$ & $\begin{array}{l}\text { ISS Debris } \\
\text { total }\end{array}$ & $\begin{array}{l}\text { ISS Debris } \\
\text { viable }\end{array}$ & $\begin{array}{l}\text { JPL-SAF } \\
\text { Debris total }\end{array}$ & $\begin{array}{l}\text { JPL-SAF } \\
\text { Debris viable }\end{array}$ & $\begin{array}{l}\text { JPL-103 } \\
\text { Debris total }\end{array}$ & $\begin{array}{l}\text { JPL-103 } \\
\text { Debris viable } \\
\end{array}$ \\
\hline \multicolumn{10}{|l|}{ Ascomycota } \\
\hline Dothideomycetes (25) & 46 & 116 & & 24 & 2681 & 2997 & 2089 & 1184 & 2362 \\
\hline Eurotiomycetes (7) & 24 & 508 & & 6278 & 1537 & 265 & 269 & 7 & 63 \\
\hline Leotiomycetes (2) & 2 & & & & & 16 & & & \\
\hline Arthoniomycetes & 8 & & & & & 51 & 29 & 1 & \\
\hline No rank & 1 & & & & & 3 & & & \\
\hline Saccharomycetes (5) & 6 & 2536 & & 2859 & & 46 & 5 & & \\
\hline Sordariomycetes (11) & 12 & & & 233 & & 66 & 34 & & \\
\hline \multicolumn{10}{|l|}{ Basidiomycota } \\
\hline Agaricomycetes (3) & 4 & & & 148 & & 8 & & & \\
\hline Cystobasidiomycetes (3) & 7 & & & & & 18 & 43 & 424 & 1020 \\
\hline Exobasidiomycetes & 6 & 498 & & 555 & & & & 3 & \\
\hline Microbotryomycetes (3) & 8 & 108 & & 401 & & 280 & 111 & 8 & \\
\hline Pucciniomycetes & 3 & & & & & 2 & 18 & & \\
\hline Tremellomycetes (5) & 23 & & & 11 & 4110 & 204 & 175 & 90 & 186 \\
\hline Chytridiomycetes & 2 & & & & & 4 & 1 & & \\
\hline Early diverging fungal lineages & 1 & & & & & 5 & 3 & & \\
\hline
\end{tabular}

The ISS HEPA filter was in service for $\sim 40$ months whereas debris collected from the ISS surface was only 1 day old (Table 1). The HEPA filter system employed in ISS was designed to revitalize the air by passing through a heat-exchange process. Hence, particulates associated with ISS HEPA filter might have been exposed to drier conditions than the crew living quarter debris (relative humidity $>50 \%$ ) and therefore the microorganisms that could withstand dry conditions only survived in the HEPA filter. Any microbial species that could withstand a prolonged period of time ( 40 months during this study) requires survival capabilities against harsh conditions, and members of the Actinobacteria phylum are known to withstand desiccation, dry conditions, and high $\mathrm{pH}[33,34]$, which explains their abundance in ISS HEPA filters ( 95.28\%; Table 3). Comparatively, sequences of viable Actinobacteria were retrieved in more numbers in ISS surface debris $(\sim 66 \%)$ than in Earth cleanroom debris $(\sim 25 \%)$ samples.

The members of Actinobacteria phylum represent human skin commensals and also soil-borne microorganisms [35]. The dominance of Actinobacteria might be due to the presence of astronauts who live and perform work activities on board the ISS. However, characterization of astronauts' skin microbiome is warranted and such studies will add more insights into the source of these bacteria associated with the ISS environmental surfaces and atmosphere. Actinobacteria have been previously reported in spacecraft assembly cleanrooms, and these non-spore-forming bacteria were considered a potential contamination risk of spacecraft due to their resistance to desiccation and ultraviolet radiation [34]. In this study, the viable population of the Actinobacteria did not constitute high population of the Earth cleanrooms, although this can be explained by the different gowning procedures required before entering these facilities unlike practiced in the ISS. In this study, both the ISS HEPA and ISS Debris samples contained high number of viable Corynebacterium sequences whereas Propionibacterium sequences were more in the ISS Debris sample. Since these microorganisms require special growth conditions, they were not isolated when traditional cultivation methods were employed during this study. Although the Propionibacterium species represent natural skin commensals, $P$. acnes is considered an opportunistic pathogen that leads to various infections $[36,37]$. Similar concerns refer to Corynebacterium, which has received attention for being a genus containing several opportunistic pathogens [38]. Even though viable sequences of these opportunistic pathogens were retrieved from both ISS locations, their virulence characteristics are to be studied before correlating their influence on human health in a closed habitat. The risk of acquiring infection from opportunistic bacterial and fungal pathogens might pose a threat to crewmembers' health and needs to be studied in the future.

In contrast, both of the Earth-based cleanrooms contained more Proteobacterial members when compared 
to the ISS locations. This trend has previously been seen in the studies of cleanroom-associated microbiomes when Sanger sequencing or DNA microarray method(s) were attempted, where human-associated Proteobacteria constituted the dominating phylum $[11,15,39]$. Low abundance ( $<100$ sequences) of Gammaproteobacteria, human commensals, in ISS samples appears reasonable since at any given time only a maximum of six astronauts are present [40]. Despite more human traffic in Earth cleanrooms, lower abundance of Gammaproteobacterial members were noticed. This might be due to the fact that appropriate countermeasures (cleaning) were in place to prevent contamination in these indoor facilities. The high abundance of Acinetobacter and Brevundimonas in the ISS Debris sample showed that these microorganisms are ubiquitous in nature and associated with the debris [41] suggesting that their presence cannot be avoided. The members of the oligotrophic class Sphingomonodaceae were more abundant in the JPL-SAF sample and were previously found to persist in these nutrient-deprived environments [42].

Among the Firmicutes, species belonging to the genus Staphylococcus were common on the ISS surfaces but not present in the ISS air. This indicates that co-inhabiting astronauts might have shed more skin microorganisms that were captured by the vacuum cleaner than the HEPA system. The cultivation-dependent method confirmed the presence of several Staphylococcus species in the ISS surface samples and these species were not isolated from other samples investigated during this study. In contrast, the higher abundance of viable Bacillus species in the JPL-SAF (Class $10 \mathrm{~K}$ ) sample suggests that they might have originated from the surrounding soil [43]. Lower abundance of Bacillus species in the more stringent JPL-103 cleanroom (Class $1 \mathrm{~K}$ ) further reiterated that appropriate maintenance could regulate microbial presence in a much cleaner indoor environment [42].

The dominant cultivable isolates of bacteria from both ISS air and ISS surfaces were spore-forming Bacillus. As members of this genus are common inhabitants of soil and dust, they were highly probable to be cultured from the ISS samples on a nutrient-rich media. When the phylogenetic affiliation results for the cultivable strains were compared to the NGS results, only Illuminaderived deep sequencing yielded Bacillus sequences. In our previous efforts, Bacillus eluded detection when traditional Sanger sequencing [44], PhyloChip G2 technology [45], or 454-pyrosequencing analysis of amplicons were employed [11]. Likewise, in this study, the pyrosequencing method again did not retrieve sequences of spore formers. In contrast, the Illumina method frequently yielded the sequences indicative of the spore formers, including members of the genus Bacillus and Paenibacillus. The absence of Solibacillus and
Brevibacillus sequences might be either attributed to the specificity of the iTag primer sets or the efficiency of the DNA extraction method employed. However, the Promega Maxwell-16 DNA extraction system used in this study was successful in extracting nucleic acids from the pure cultures of Solibacillus and Brevibacillus. Despite the same sample preparation, the Illumina-based sequencing generated more reads (40-2400 times; average $\sim 100$ times), thus providing a deeper coverage of microbial diversity than the 454 platform. Choosing an appropriate technology is important when abundance and richness of the microbial diversity are considered. Although Illumina can be employed to understand yetto-be cultivated microorganisms, the use of wellestablished traditional techniques should not be completely abandoned.

Fungal strains have been detected on the ISS multiple times as actively growing molds on the environmental surfaces or responsible microorganisms deteriorating the components of ISS hardware [4]. The abundance of the fungal molds in indoor environments was reported to be associated with invasive infections and allergies [46, 47]. Therefore, a high number of fungal isolates from the ISS locations confirmed a need for continuous monitoring of the ISS for fungal populations. Aspergillus niger was the predominant isolate, and although it does not have the potential to cause disease at the same rate as other Aspergillus species (A. fumigatus, A. flavus), it was correlated with pulmonary and ear infections [48]. Aspergillus species are repeatedly isolated in indoor microbiomes [49]. The detection of multiple isolates of $A$. niger is consistent with its frequent presence in built environments including ISS surfaces [50]. However, its survival capability, proliferation, and molecular alteration in response to ISS microgravity conditions need to be studied further. On the other hand, A. niger is known for production of many beneficial substances. The potential for production of novel beneficial secondary metabolites under microgravity conditions needs to be explored for use in diverse biotechnological and pharmaceutical fields $[51,52]$.

\section{Conclusions}

This study provided important insight into the ISS microbial diversity with a focus on significant differences between the closed habitats of the ISS and Earth-based cleanrooms. The NGS data, supported by traditional microbiology techniques and selective molecular bioburden estimation assays, provided a preliminary but detailed report on the ISS environmental microbiome. Future work will be able to build on these initial results by determining how stable that environment is over time. In contrast with the JPL cleanrooms, the most common organisms in the ISS environment were 
members of the Actinobacteria that are frequently associated with humans. As on the ISS, human traffic is common in the cleanrooms, but clearly the more stringent procedures possible on Earth are more effective in eradicating these organisms. The current results provide a baseline for evaluating the effectiveness of future ISS procedural changes. This study also allowed an evaluation of the utility of coupling PMA treatment with NGS sequencing for more accurate estimation of viable population. The bacterial diversity of each sample decreased after the PMA treatment but this was strongly associated with the sampling site. Since exposure to viable organisms is best minimized, it is important to know which areas are most problematic and hence should be avoided and/or targeted for more stringent cleaning conditions. It is clear from the results presented here that viable cell studies with PMA can provide this information. It was also found that the relative numbers of various types of viable cells in each sample closely resembled the distribution obtained when all (dead and viable) cells were considered. This is likely to be a usual result unless the population at a sampling site has been recently and dramatically changed such that the prior dominant organisms have been replaced in only the pool of viable cells.

\section{Methods}

\section{Sample characteristics}

Particulate materials were collected from the ISS HEPA filter element (ISS HEPA) and vacuum cleaner bag components (ISS Debris) used aboard the ISS as well as at two cleanrooms at JPL (one each originating from the SAF and building 103, Pasadena, CA), during this study. Table 1 lists sample characteristics, usage time of the material collection devices or system(s), model, make, and cleanroom conditions where the devices were used. The materials collected using the HEPA filter system represented the air and the vacuum cleaner bag represented the surface locations.

\section{ISS HEPA}

The environmental control system (ECS) aboard the ISS includes a distributed ventilation system that contains HEPA filter elements to remove suspended particulate matter from the cabin atmosphere and protect humidity control and air purification equipment from debris accumulation and biofouling [53]. Particle counts are not routinely measured aboard crewed spacecraft. A study conducted during space shuttle mission STS-32 (January 1990) found that the particle size distribution approximated a class 100,000 cleanroom for particles $<2.5 \mu \mathrm{m}$, a class 400,000 cleanroom for particles $2.5 \mu \mathrm{m}$ to $<10 \mu \mathrm{m}$, and a class 3000 cleanroom for particles $>10 \mu \mathrm{m}[53,54]$. The particle size distributions are defined using cleanroom standard definitions (FED-STD209 1992). The HEPA filters used aboard the ISS retain $99.97 \%$ particles of $0.3 \mu \mathrm{m}$ via a pleated borosilicate media. A 20-mesh $(841-\mu \mathrm{m}$ sieve openings) inlet screen located at the filter element's face removes larger debris and fibers. The pleated, non-woven borosilicate HEPA filter media was installed by Flanders Filters, Inc. (Washington, NC) in the filter element housing. Flanders Filters, Inc.'s commercial "NaturalAire" cut-to-fit filtration media has the greatest similarity to the ISS HEPA filter media (http://flandersfilters.com/products/naturalaire/). Twenty-one filter elements are distributed throughout the ISS in several modules and astronauts replace them on a scheduled maintenance cycle ranging from 2.5 to 5 years, depending on the location. The part number of the HEPA filter system analyzed during this study was SV810010-1 and the serial number was 0049. The HEPA filter element analyzed during this study was manufactured in September 1998, installed in ISS on January 2008, and returned aboard space shuttle flight STS-134/ULF6 in late May 2011. This filter was installed in ISS Node 2 and was in service for 40 months, although the typical service time is only 30 months for this location. Typically, ground testing is performed to characterize a filter element's pressure drop at various process air flow rates [53] after retrieval from the ISS, but in this case, the HEPA filter remained untouched in its shipment packaging from the time it was removed from the ISS until particulates were recovered at JPL for microbial characterization. The particulate materials collected from this sample were designated as ISS HEPA during this study.

\section{ISS debris}

The vacuum cleaner bag contained debris that had collected on the HEPA filter inlet screens. The vacuum cleaner bag components are representative of the collected debris (lint, food particles, skin/hair, and miscellaneous debris) that had been airborne in the cabin but had collected over time on the filter element inlet screen. The molecular microbial community analysis for the ISS vacuum bag debris (ISS Debris) was previously documented [14]. These results were utilized here for comparative purposes. The previous molecular characterizations of the ISS Debris sample were extended here with Illumina-based deep sequencing using archived DNA aliquots [14]. Sample characteristics of the ISS Debris are described in Table 1. Briefly, during the period of ISS Expeditions 30 and 31, reports by some crewmembers cited differences in the cabin environment compared to earlier experience as well as allergic responses to the cabin environment. One of the noted observations was a high level of visible dust in the ISS 
Node 3 cabin, to the extent it was sticking to the walls. Flight surgeons indicated that this had been reported not just in Node 3 but also throughout the US on-orbit segment and expressed a concern for crew health. Dust on the ISS is expected, with humans being major contributors (via skin shedding, eating, exercising, etc.). Other sources such as on-orbit maintenance activities can release dust from sources such as payloads and systems, clothing, and visiting vehicles. As a precautionary measure, in the middle of 2012, an investigation was launched to define and mitigate dust sources and to determine if exposure to dust might elicit an adverse effect on crew health. As a result of these crewmember reports, particulate and fiber debris samples were collected during ISS Expedition 31 using a handheld portable vacuum cleaner and returned to Earth aboard Soyuz flight $29 \mathrm{~S}$ in early July 2012 for analyses. Details of the novel "Prime" vacuum cleaner developed for NASA habitats were reported elsewhere [55]. Portions of the samples were aseptically collected for microbiological testing at NASA's Johnson Space Center (JSC), with the remaining bag and its contents repacked in a biological hood, sealed, and shipped to NASA's Marshall Space Flight Center (MSFC) for particle size testing, as well as to JPL to determine their molecular microbiological composition. The vacuum cleaner bag was shipped via FedEx at room temperature with instructions that the samples not be irradiated in transport.

\section{Earth analogs}

For comparison, particles collected from two JPL cleanroom floors using a Nilfisk GM80CR vacuum cleaner (the disposable bag part number is 81620000 ; Morgantown, PA) were analyzed (Table 1). Cleanroom certification is based on the maximum number of particles greater than $0.5 \mu \mathrm{m}$ per cubic foot of air. The air within Class $1 \mathrm{~K}$ cleanrooms is maintained at fewer than 1000 particulates per cubic foot, Class $10 \mathrm{~K}$ cleanrooms are allowed to harbor a density of 10,000 particles per cubic foot, and so on. The cleanroom samples were from (a) the JPL-SAF cleanroom floor where various Mars mission spacecraft were built (Class $10 \mathrm{~K}$; JPL-SAF Debris) and (b) JPL building 103 where non-mission critical activities were conducted (Class $1 \mathrm{~K}$; JPL-103 Debris). The vacuum cleaner bag debris from the JPL-SAF and JPL-103 buildings was transported to the microbiology laboratories for storage. Sample processing was carried out immediately after aseptic collection of the materials, usually within 7 days from the retrieval of the vacuum bags from the cleanrooms. Both Earth-analog samples were processed at the same time as the ISS Debris samples. However, the ISS HEPA sample was received $\sim 3$ months later and hence was analyzed separately.

\section{Sample processing}

All samples were subjected to a variety of microbiological and molecular techniques to elucidate composition of cultivable, viable, and total microorganisms. Independent of the samples taken to cultivate bacterial and fungal analyses, subsamples of the same samples were taken for DNA extraction. Weighing vacuum debris samples was possible, whereas the HEPA filter elements were divided into small pieces and particulates associated with the pieces were aseptically collected using sterile scalpels before being quantitatively measured. Approximately $1 \mathrm{~g}$ of each vacuum debris and HEPA filter associated particle was weighed and placed into a sterile tube containing $25 \mathrm{~mL}$ of sterile phosphate-buffered saline (PBS) and vortexed for $1 \mathrm{~min}$. After vigorous mixing, large particles were allowed to settle, and aliquots of samples were carefully siphoned and allocated for culture-based $(1 \mathrm{~mL})$ and cultureindependent analyses $(15 \mathrm{~mL})$.

\section{Traditional culture-based microbial examination}

For estimating bacterial populations, after suitable serial tenfold dilution in sterile PBS, $100 \mu \mathrm{L}$ of the sample suspension was spread onto two plates of R2A media (BD Difco, Franklin Lakes, NJ) and incubated at $25{ }^{\circ} \mathrm{C}$ for $2-$ 7 days. For the fungal population enumeration, $100 \mu \mathrm{L}$ was spread onto two plates of potato dextrose agar (PDA, BD Difco, Franklin Lakes, NJ) and incubated at $25{ }^{\circ} \mathrm{C}$ for $2-$ 7 days. Bacterial and fungal colony-forming units (CFUs) were counted and reported as CFU/g of material. Identifications and phylogenetic affiliations were carried out via sequencing for both bacteria and fungi by targeting the $16 \mathrm{~S}$ rRNA gene [42] and the ITS region [56], respectively. When identifications were ambiguous, sequencing of an additional gene $(\mathrm{gyr} B)$ was performed to confirm the phylogenetic identity of the purified bacterial isolates [57, 58]. The nucleotide sequences of bacteria (KT763339KT763368) and fungi (KT832780-KT832790) were deposited in GenBank.

\section{Sample processing for molecular analysis}

The biological materials associated with each sample $(15 \mathrm{~mL})$ were further concentrated using Amicon Ultra-50 Ultracel centrifugal filter tubes (Millipore, Billerica, MA). Each filter unit has a molecular mass cutoff of $50 \mathrm{kDa}$, which facilitates the concentration of microbial cells, spores, and exogenous nucleic acid fragments greater than $100 \mathrm{bp}$ in a final volume of $2.5 \mathrm{~mL}$. All filtered samples were then divided into three separate aliquots: the first aliquot $(1000 \mu \mathrm{L})$ was subjected to PMA pretreatment (viability assessment), the second $(1000 \mu \mathrm{L})$ was an untreated 
environmental sample (viable + nonviable; total DNA), and the third $(500 \mu \mathrm{L})$ was used for adenosine triphosphate (ATP) analysis (see below).

For measuring viable microbial population, one aliquot of filter-concentrated sample suspension $(1000 \mu \mathrm{L})$ was treated with $12.5 \mu \mathrm{L}$ of PMA (2 mM; Biotium, Inc., Hayward, CA) to a final concentration of $25 \mu \mathrm{M}$ [26, 32], followed by thorough mixing and incubation in the dark for $5 \mathrm{~min}$ at room temperature [15]. The sample was exposed to PhAST blue-Photo activation system for tubes (GenIUL, S.L., Terrassa, Spain) for $15 \mathrm{~min}$ (in parallel with the non-PMA-treated sample). This step enabled blocking DNA from dead cells [15]. The samples were then split in half and one half was subjected to bead beating with the Fastprep-24 bead-beating instrument (MP Biomedicals, Santa Ana, CA) with parameters set at $5 \mathrm{~m} / \mathrm{s}$ for $60 \mathrm{~s}$. The second half of the unprocessed sample was then combined with the mechanically disrupted counterpart before DNA was extracted via the Maxwell 16 automated system (Promega, Madison, WI), in accordance with manufacture instructions [59]. Resulting DNA suspensions (100 $\mu \mathrm{L}$ each) were stored at $-20^{\circ} \mathrm{C}$.

\section{Quantitation of total and viable microorganisms using molecular methods \\ ATP assay}

A bioluminescence assay was performed to determine the total ATP and intracellular ATP from all samples using the CheckLite HS kit (Kikkoman Corp., Noda, Japan), as described previously [60]. Briefly, to determine the total ATP (dead and viable microbes), $100-\mu \mathrm{L}$ sample aliquots (four replicates) were each combined with $100 \mu \mathrm{L}$ of a cell lysing detergent (benzalkonium chloride) then incubated at room temperature for $1 \mathrm{~min}$ prior to the addition of $100 \mu \mathrm{L}$ of a luciferin-luciferase reagent. The sample was mixed, and the resulting bioluminescence was measured with a luminometer, the Lumitester K-210 (Kikkoman Corp.). To determine intracellular ATP (viable microbes), $50 \mu \mathrm{L}$ of an ATP-eliminating reagent (apyrase, adenosine deaminase) was added to a $500-\mu \mathrm{L}$ portion of the sample and allowed to incubate for $30 \mathrm{~min}$ to remove any extracellular ATP, after which the assay for ATP was carried out, as described above, in four replicates, including sterile PBS as negative controls. As previously established, one RLU, the unit of measurement of ATP, was assumed to be approximately equal to one CFU [42].

\section{qPCR assay}

Real-time quantitative polymerase chain reaction (qPCR) assay, targeting the $16 \mathrm{~S}$ rRNA gene, was performed in triplicate with a CFX-96 thermal cycling Instrument (Bio-Rad, Hercules, CA) to quantify the bacterial burden. Bacteria-directed primers targeting the $16 \mathrm{~S}$ rRNA gene 1369 F (5'-CGG TGA ATACGT TCY CGG-3') and modified 1492R (5'-GGW TAC CTTGTT ACG ACT T-3') were used for this analysis [61]. Each $25-\mu \mathrm{L}$ reaction consisted of $12.5 \mu \mathrm{L}$ of $2 \mathrm{X}$ iQ SYBR Green Supermix (BioRad, Hercules, CA), $1 \mu \mathrm{L}$ each of forward and reverse oligonucleotide primers (10 $\mu \mathrm{M}$ each), and $1 \mu \mathrm{L}$ of template DNA. Purified DNA from B. pumilus cells served as the positive control and DNase/RNasefree molecular-grade distilled water (UltraPure, Gibco) was used as the negative control. These controls were included in all qPCR runs. Reaction conditions were as follows: a 3-min denaturation at $95{ }^{\circ} \mathrm{C}$, followed by 35 cycles of denaturation at $95{ }^{\circ} \mathrm{C}$ for $15 \mathrm{~s}$, and a combined annealing and extension at $55^{\circ} \mathrm{C}$ for $35 \mathrm{~s}$.

\section{Molecular microbial diversity analysis using next-generation sequencing methods Pyrosequencing conditions}

Bacterial primers 28 F (5'-GAG TTT GAT CNT GGC TCA G-3') and 519R (5'-GTN TTA CNG CGG CKG CTG-3') were used to amplify 500-bp fragments spanning the V1-V3 hypervariable regions of the bacterial $16 \mathrm{~S}$ rRNA gene. Archaeal primers $341 \mathrm{~F}$ (5'-GYG CAS CAG KCG MGA AW-3') and 958R (5'-GGA CTA CVS GGG TAT CTA AT-3') were used to amplify 600-bp fragments spanning the V3-V5 hypervariable regions of the archaeal 16S rRNA gene. A fungal primer set ITS1F (5'-CTT GGT CAT TTA GAG GAA GTA A-3') and ITS4R (5' -TCC TCC GCT TAT TGA TAT GC-3') was employed to amplify $\sim 600$-bp fragments of the fungal ITS region. These primer pairs were tailored for pyrosequencing by adding a fusion linker and a proprietary 8 -nt barcode sequence at the $5^{\prime}$ end of the forward primer and a biotin and fusion linker sequence at the $5^{\prime}$ end of the reverse primer [62]. A HotStarTaq Plus master mix kit (Qiagen, Valencia, CA) was used to catalyze the PCR under the following thermal cycling conditions: initial denaturing at $95^{\circ} \mathrm{C}$ for $5 \mathrm{~min}$, followed by 35 cycles of denaturing at $95{ }^{\circ} \mathrm{C}$ for $30 \mathrm{~s}$, annealing at $54{ }^{\circ} \mathrm{C}$ for $40 \mathrm{~s}$, and extension at $72{ }^{\circ} \mathrm{C}$ for $1 \mathrm{~min}$, finalized by a 10 -min elongation at $72{ }^{\circ} \mathrm{C}$. Resulting PCR products were purified via Diffinity Rapid Tip (Diffinity Genomics, Inc., West Henrietta, NY) chemistry and were then pooled accordingly. Small fragments were removed with Agencourt Ampure Beads (Beckman Coulter, Brea, CA).

In preparation for FLX-Titanium sequencing (Roche, Nutley, NJ), resulting PCR amplicon fragment size and concentration were accurately measured with DNA 1000 chips using a Bioanalyzer 2100 automated electrophoresis station (Agilent, Santa Clara, CA) and a TBS-380 Fluorometer (Turner Biosystems, Sunnyvale, CA). The total volume of initial PCR product used for subsequent emulsion PCR was $2 \mu \mathrm{L}$ for strong positives $(>10 \mathrm{ng} / \mu \mathrm{L})$, $5 \mu \mathrm{L}$ for weak positives ( 5 to $10 \mathrm{ng} / \mu \mathrm{L}$ ), and $20 \mu \mathrm{L}$ for samples that failed to yield PCR products $(<5 \mathrm{ng} / \mu \mathrm{L})$. 
This normalization step helped to ensure minimal bias favoring downstream amplification from initially strong PCR products. Approximately $9.6 \times 10^{6}$ molecules of 600-bp double-stranded DNA were combined with $9.6 \times 10^{6}$ DNA capture beads and then subjected to emulsion PCR conditions. Following recovery and enrichment, bead-attached DNA molecules were denatured with $\mathrm{NaOH}$ and sequencing primers were annealed. A four-region 454 pyrosequencing run was performed on a GS PicoTiterPlate (PTP) using the Genome Sequencer FLX System, in accordance with manufacturer instructions (Roche, Nutley, NJ). Twenty-four to 30 tagged samples were applied to each quarter region of the PTP. All pyrosequencing procedures were performed at the Research and Testing Laboratory (Lubbock, TX) in accordance with well-established protocols [62].

\section{Illumina sequencing conditions}

The library preparations for next-generation sequencing and Illumina MiSeq sequencing were conducted by GENEWIZ, Inc. (South Plainfield, NJ). The DNA samples were quantified using a Qubit 2.0 fluorometer (Invitrogen, Carlsbad, CA) and DNA quality was confirmed by electrophoresis (0.8\% agarose gel). The sequencing library was constructed using a MetaVx ${ }^{\mathrm{Ts}}$ Library Preparation kit (GENEWIZ, Inc., South Plainfield, NJ). In brief, 5-50 ng of DNA was used for amplicon generation to cover the V3 and V4 hypervariable regions of $16 \mathrm{~S}$ rDNA. Indexed and universal adapters were added to the ends of the $16 \mathrm{~S}$ rDNA amplicons by limited-cycle PCR. The DNA libraries were validated with an Agilent 2100 Bioanalyzer (Agilent Technologies, Palo Alto, CA) and quantified using Qubit and real-time PCR (Applied Biosystems, Carlsbad, CA). The DNA libraries were multiplexed and loaded on an Illumina MiSeq following the instructions from the manufacturer (Illumina, San Diego, CA). A $2 \times 150$ paired-end (PE) configuration was used for sequencing. The image analysis and base calling were processed using MiSeq Control Software. The initial taxonomy was performed on Illumina's BaseSpace cloud computing platform.

\section{Bioinformatic analyses of bacterial pyrosequences}

High-throughput $16 \mathrm{~S}$ rRNA sequencing data were processed. Bacterial and archaeal sequences were processed and analyzed using the mothur software [16, 63]. Sequences were quality filtered by removing sequences that (a) did not contain the primer sequence, (b) contained an uncorrectable barcode, (c) were <250 nt in length, (d) had homopolymers longer than $8 \mathrm{nt}$, or (e) had a quality score of $<25$; and then demultiplexed using the respective sample nucleotide barcodes. These sequences were searched against the Greengenes reference database $[64,65]$ and clustered into OTUs based on their sequence similarity
(97 \%) with UCLUST [66]. A representative sequence was picked from each OTU and taxonomic classification was assigned using mothur's Bayesian classifier [67] and Greengenes training sequences and taxonomy [64, 65].

\section{Bioinformatic analyses of fungal pyrosequences}

The sequences were run through ITSx 1.0.9 [68] to remove non-ITS sequences, assembly chimeras, and sequences for which none of the 3 ' end of SSU rDNA or the $5^{\prime}$ end of $5.8 \mathrm{~S}$ rDNA was detected. The ITS1 subregion was extracted from the remaining sequences for further analyses. Chimera control was exercised through UCHIME 7 [69] using the UNITE chimera reference dataset [70] as reference corpus. The sequences were subjected to clustering and taxonomic assignment in the Sequence Clustering and Analysis of Tagged Amplicons (SCATA) next-generation sequencing analysis pipeline (http://scata.mykopat.slu.se) using the February 2014 release of UNITE as taxonomic reference corpus. The default sequence quality control settings of SCATA were used; however, the clustering threshold was set to $98.5 \%$ [71]. All taxonomic affiliations proposed by SCATA were examined manually using Basic Local Alignment Search Tool (BLAST) 2.2.30 [72] in GenBank [73] and UNITE [74] and occasionally refined using the principles outlined in Koljalg et al. [75] and Nilsson et al. [76].

\section{Preprocessing of bacterial Illumina sequences}

The 12 sets of $16 \mathrm{~S}$ rDNA V3 amplicon data were sequenced at GENEWIZ, Inc. (South Plainfield, NJ), where the obtained reads were trimmed to remove primer sequences. The raw sequence dataset was composed of $10,241,173$ paired-end reads, 150 bp in length, with exceptionally high quality $(<0.1 \%$ error rate) (Resphera Discovery $^{\mathrm{TM}}$ protocol, Baltimore, MD). After trimming noisy reads and removal of low-quality and chimeric sequences, we identified a moderate level of expected contaminants, including chloroplast and mammalian mitochondrial sequence (range 0.0001-9.3\%), as well as a low level of unknown contaminants (range 0.01-1.8 \%). Exploratory characterization of unknown contaminant representatives indicated nonspecific amplification mitochondrial sequences from various eukaryotic organisms. After completion of preprocessing, the resulting high-quality $\mathrm{R} 1$ and $\mathrm{R} 2$ read sets contained 9.1 $\mathrm{M}$ and 9.3 $\mathrm{M}$ sequences, respectively, with an average length of $128 \mathrm{bp}$. Due to the primer set and sequencing technology selected by the vendor, we were unable to merge paired-end sequences into longer consensus fragments as they did not overlap. Therefore, in this analysis, we compared our findings between the R1 (forward)- and R2 (reverse)-associated datasets to evaluate consistency. Across R1 and R2 datasets, each sample had on average 917,060 clean sequences. After clustering sequences into OTUs, OTU tables were 
rarefied to an even coverage of 525,000 sequences per sample. On average, Good's coverage statistic for rarefied samples was $99.78 \%$, indicating we have observed the vast majority of OTU diversity in each community.

\section{Bioinformatic analysis of bacterial Illumina sequences}

Sequences were de-multiplexed using $5^{\prime}$ barcode identifiers and analyzed using the Resphera Discovery ${ }^{\text {max }}$ protocol (Baltimore, MD). Briefly, 16S rRNA sequence fragments were first screened in Quantitative Insights into Microbial Ecology (QIIME) [77] for quality and length requiring: (a) trimming at the first 5-bp run of Phred quality scores below 20, (b) one ambiguous base call or less, and (c) a minimum final length of $125 \mathrm{bp}$ after trimming of forward and reverse primer sequences. Passing sequences were screened for PhiX-174 spiked fragments and PCR-associated chimeras by UCHIME [69] (de novo mode). Non-chimeric reads were then filtered for contaminant chloroplast and mitochondrial sequences using the RDP classifier [17], followed by a broad nucleotide BLAST (BLASTN) [78] search of the GreenGenes 16S rRNA reference database [65] (v1.1) to identify potential unknown contaminants. The resulting high-quality dataset was clustered into de novo OTUs using UCLUST [66] with a $95 \%$ identity threshold. OTU representatives were assigned to a taxonomic lineage using the RDP classifier trained on the Resphera 16S rRNA database (v1.1) requiring a minimum confidence score of 0.50 .

\section{Statistical analyses}

To perform pairwise statistical comparisons of sample groups of interest, a negative binomial test implemented in DESeq [79] or Fisher's exact test was utilized and controlled for false positives using the false discovery rate (FDR) [80]. For the comparison of bacterial pyrosequence and Illumina data and fungal pyrosequence analysis, multivariate statistical analyses of community profiles were performed using an "in-house R-script" employing the libraries vegan, ape, gplots, mgcv, and GUniFrac [81]. First, each dataset composed of OTU abundances per sample was rarefied to the lowest number of reads and a Bray-Curtis index was calculated. This procedure was repeated 10,000 times and the average Bray-Curtis distance was calculated for each dataset in order to avoid biases arising from rarefication. The Bray-Curtis distance was then utilized to calculate principal coordinate (PCoA) analyses, PERMANOVA (1000 permutations), and MRPP (1000 permutations).

\section{Availability of supporting data}

The data set supporting the results of this article is available in the NCBI SRA repository, under SRA \#280254.

\section{Additional files}

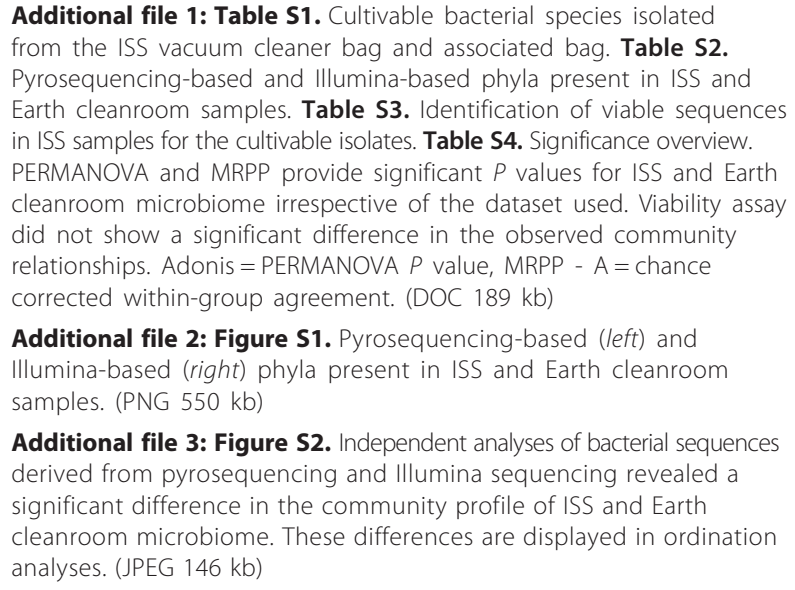

Additional file 2: Figure S1. Pyrosequencing-based (left) and Illumina-based (right) phyla present in ISS and Earth cleanroom samples. (PNG $550 \mathrm{~kb}$ )

Additional file 3: Figure S2. Independent analyses of bacterial sequences derived from pyrosequencing and Illumina sequencing revealed a significant difference in the community profile of ISS and Earth cleanroom microbiome. These differences are displayed in ordination analyses. (JPEG $146 \mathrm{~kb}$ )

\section{Competing interests}

The authors declare that they have no competing interests.

\section{Authors' contributions}

AC drafted the manuscript, contributed to the data analysis and interpretation, and carried out the identification of the isolates in this study. AJP contributed to the statistical analysis and data interpretation. JRW performed the statistical analysis of Illumina data. PV performed the experimental part of the study (cultivation and molecular biology based studies). DK, VGS, and GEF helped with the bioinformatics. HRN analyzed the fungal sequence dataset. DLP helped to analyze ISS Debris samples. JP procured the ISS samples and helped with the study concept and design. KV designed the study, interpreted the data, and drafted the manuscript. All authors read and approved the final manuscript.

\section{Acknowledgements}

Part of the research described in this publication was carried out at the Jet Propulsion Laboratory, California Institute of Technology, under a contract with NASA. This research was funded by a 2012 Space Biology NNH12ZTT001N grant no. 19-12829-26 under Task Order NNN13D111T award to K. Venkateswaran, (c) 2015 California Institute of Technology. Government sponsorship acknowledged.

\section{Author details}

${ }^{1}$ Jet Propulsion Laboratory, Biotechnology and Planetary Protection Group, California Institute of Technology, M/S 89-2 4800 Oak Grove Dr., Pasadena, CA 91109, USA. ²Department of Earth and Planetary Sciences, University of California, Berkeley, CA, USA. ${ }^{3}$ Resphera Biosciences, Baltimore, MD, USA. ${ }^{4}$ Department of Biology and Biochemistry, University of Houston, Houston, TX, USA. ${ }^{5}$ Department of Biological and Environmental Sciences, University of Gothenburg, Gothenburg, Sweden. ${ }^{6} J o h n s o n$ Space Center, Houston, TX, USA. ${ }^{7}$ Marshall Space Flight Center, Huntsville, AL, USA.

Received: 28 July 2015 Accepted: 28 September 2015

Published online: 27 October 2015

\section{References}

1. Castro VA, Thrasher AN, Healy M, Ott CM, Pierson DL. Microbial characterization during the early habitation of the International Space Station. Microb Ecol. 2004;47(2):119-26.

2. La Duc MT, Pierson DL, Venkateswaran K. Evidence of pathogenic microbes in the International Space Station drinking water: reason for concern? Habitation. 2004;10:39-48.

3. Vesper SJ, Wong W, Kuo CM, Pierson DL. Mold species in dust from the International Space Station identified and quantified by mold-specific quantitative PCR. Res Microbiol. 2008;159(6):432-5. doi:10.1016/ j.resmic.2008.06.001. 
4. Novikova N, De Boever P, Poddubko S, Deshevaya E, Polikarpov N, Rakova N, et al. Survey of environmental biocontamination on board the International Space Station. Res Microbiol. 2006;157(1):5-12

5. Satoh K, Nishiyama Y, Yamazaki T, Sugita T, Tsukii Y, Takatori K, et al. Microbe-l: fungal biota analyses of the Japanese experimental module KIBO of the International Space Station before launch and after being in orbit for about 460 days. Microbiol Immunol. 2011;55(12):823-9. doi:10.1111/j.1348-0421.2011.00386.x

6. Kelley ST, Gilbert JA. Studying the microbiology of the indoor environment Genome Biol. 2013;14:202.

7. NRC. Committee for the Decadal Survey on Biological Physical Sciences in Space: recapturing a future for space exploration: life and physical sciences research for a new era. Washington, DC: The National Academies Press; 2011.

8. Crabbe A, Schurr MJ, Monsieurs P, Morici L, Schurr J, Wilson JW, et al. Transcriptional and proteomic responses of Pseudomonas aeruginosa PAO1 to spaceflight conditions involve Hfq regulation and reveal a role for oxygen. Appl Environ Microbiol. 2011;77(4):1221-30. doi:10.1128/AEM.01582-10.

9. Wilson JW, Ott CM, Honer zu Bentrup K, Ramamurthy R, Quick L, Porwollik S, et al. Space flight alters bacterial gene expression and virulence and reveals a role for global regulator Hfq. Proc Natl Acad Sci U S A. 2007;104(41):16299-304. doi:10.1073/pnas.0707155104.

10. Yergeau E, Lawrence JR, Sanschagrin S, Waiser MJ, Korber DR, Greer CW. Next-generation sequencing of microbial ocmmunities in the Athabasca River and its tributaries in relation to oil sands mining activities. Appl Environ Microbiol. 2012;78(21):7626-37.

11. La Duc MT, Vaishampayan P, Nilsson HR, Torok T, Venkateswaran K. Pyrosequencing-derived bacterial, archaeal, and fungal diversity of spacecraft hardware destined for Mars. Appl Environ Microbiol. 2012;78(16):5912-22. doi:10.1128/AEM.01435-12.

12. Human Microbiome Project C. Structure, function and diversity of the healthy human microbiome. Nature. 2012;486(7402):207-14. doi:10.1038/nature11234

13. Lazarevic V, Whiteson $K$, Huse $S$, Hernandez D, Farinelli L, Østerås $M$, et al. Metagenomic study of the oral microbiota by Illumina high-throughput sequencing. J Microbiol Methods. 2009;79:266-71.

14. Venkateswaran K, Vaishampayan P, Cisneros J, Pierson DL, Rogers SO, Perry J. International Space Station environmental microbiome-microbial inventories of ISS filter debris. Appl Microbiol Biotechnol. 2014;98(14):6453-66. doi:10.1007/ s00253-014-5650-6.

15. Vaishampayan P, Probst AJ, La Duc MT, Bargoma E, Benardini JN, Andersen $\mathrm{GL}$ et al. New perspectives on viable microbial communities in low-biomass cleanroom environments. ISME J. 2013;7(2):312-24. http://www.nature.com/ ismej/journal/v7/n2/suppinfo/ismej2012114s1.html.

16. Schloss PD, Westcott SL, Ryabin T, Hall JR, Hartmann M, Hollister EB, et al Introducing mothur: open-source, platform-independent, communitysupported software for describing and comparing microbial communities. Appl Environ Microbiol. 2009;75(23):7537-41. doi:10.1128/AEM.01541-09.

17. Wang Q, Garrity GM, Tiedje JM, Cole JR. Naïve Bayesian classifier for rapid assignment of rRNA sequences into the new bacterial taxonomy. Appl Environ Microbiol. 2007;73(16):5261-7. doi:10.1128/aem.00062-07.

18. Cabral JPS. Can we use indoor fungi as bioindicators of indoor air quality? Historical perspectives and open questions. Sci Total Environ. 2010;408(20):4285-95. http://dx.doi.org/10.1016/j.scitotenv.2010.07.005.

19. Cooley JD, Wong WC, Jumper CA, Straus DC. Correlation between the prevalence of certain fungi and sick building syndrome. Occup Environ Med. 1998:55:579-84.

20. Favero MS. Microbiologic assay of space hardware. Environ Biol Med. 1971;1(1):27-36

21. Favero MS, Puleo JR, Marshall JH, Oxborrow GS. Comparative levels and types of microbial contamination detected in industrial clean rooms. Appl Microbiol. 1966;14(4):539-51.

22. Favero MS, Puleo JR, Marshall JH, Oxborrow GS. Comparison of microbial contamination levels among hospital operating rooms and industrial clean rooms. Appl Microbiol. 1968;16(3):480-6.

23. La Duc MT, Kern RG, Venkateswaran K. Microbial monitoring of spacecraft and associated environments. Microb Ecol. 2004;47:150-8.

24. Venkateswaran K, La Duc MT, Vaishampayan P. Genetic Inventory Task: Final Report, JPL Publication 12-12. Pasadena, CA: Jet Propulsion Laboratory, California Institute of Technology; 2012. p. 1-117.

25. Nocker A, Camper AK. Novel approaches toward preferential detection of viable cells using nucleic acid amplification techniques. FEMS Microbiol Lett. 2009;291(2):137-42. doi:10.1111/j.1574-6968.2008.01429.x.
26. Nocker A, Richter-Heitmann T, Montijn R, Schuren F, Kort R. Discrimination between live and dead cellsin bacterial communities from environmental water samples analyzed by 454 pyrosequencing. Int Microbiol. 2010;13(2):59-65.

27. Nocker A, Sossa-Fernandez P, Burr MD, Camper AK. Use of propidium monoazide for live/dead distinction in microbial ecology. Appl Environ Microbiol. 2007;73(16):5111-7. doi:10.1128/AEM.02987-06.

28. La Duc MT, Venkateswaran K, Conley CA. A genetic inventory of spacecraft and associated surfaces. Astrobiology. 2014;14(1):15-23. doi:10.1089/ast.2013.0966.

29. Mahnert A, Vaishampayan P, Probst AJ, Auerbach A, Moissl-Eichinger C, Venkateswaran $\mathrm{K}$, et al. Cleanroom maintenance significantly reduces abundance but not diversity of indoor microbiomes. PLoS One. 2015;10(8), e0134848. doi:10.1371/journal.pone.0134848.

30. Salter SJ, Cox MJ, Turek EM, Calus ST, Cookson WO, Moffatt MF, et al. Reagent and laboratory contamination can critically impact sequence-based microbiome analyses. BMC Biol. 2014;12(1):87. doi:10.1186/s12915-014-0087-z.

31. Laurence M, Hatzis C, Brash DE. Common contaminants in next-generation sequencing that hinder discovery of low-abundance microbes. PLoS One. 2014;9(5), e97876. doi:10.1371/journal.pone.0097876.

32. Rawsthorne $H$, Dock CN, Jaykus LA. PCR-based method using propidium monoazide to distinguish viable from nonviable Bacillus subtilis spores. Appl Environ Microbiol. 2009;75(9):2936-9. doi:10.1128/AEM.02524-08.

33. Kieft TL. Hot desert soil communities. In: Bitton G, editor. Encyclopedia of Environmental Microbiology. NY: John Wiley; 2002. p. 1576-86.

34. Osman S, Peeters Z, La Duc MT, Mancinelli R, Ehrenfreund P, Venkateswaran K. Effect of shadowing on survival of bacteria under conditions simulating the Martian atmosphere and UV radiation. Appl Environ Microbiol. 2008;74(4):959-70. doi:10.1128/AEM.01973-07.

35. Ventura M, Canchaya C, Tauch A, Chandra G, Fitzgerald GF, Chater KF, et al. Genomics of Actinobacteria: tracing the evolutionary history of an ancient phylum. Microbiol Mol Biol Rev. 2007;71(3):495-548. doi:10.1128/ mmbr.00005-07.

36. McDowell A, Valanne S, Ramage G, Tunney MM, Glenn JV, McLorinan GC, et al. Propionibacterium acnes types I and II represent phylogenetically distinct group. J Clin Microbiol. 2005;43(1):326-44.

37. Achermann Y, Goldstein EJC, Coenye T, Shirtliff ME. Propionibacterium acnes: from commensals to opportunistic biofilm-associated implant pathogen. Clin Microbiol Rev. 2014;27(3):419-40.

38. Bernard K. The genus Corynebacterium and other medically relevant coryneform-like bacteria. J Clin Microbiol. 2012;50(10):3152.

39. Moissl C, Osman S, La Duc MT, Dekas A, Brodie E, DeSantis T, et al. Molecular bacterial community analysis of clean rooms where spacecraft are assembled. FEMS Microbiol Ecol. 2007;61(3):509-21. doi:10.1111/j.1574-6941.2007.00360.x.

40. Thirsk R, Kuipers A, Mukai C, Williams D. The space-flight environment: the International Space Station and beyond. Can Med Assoc J. 2009:180(12):1216-20.

41. Vaishampayan P, Osman S, Andersen G, Venkateswaran K. High-density $16 S$ microarray and clone library-based microbial community composition of the Phoenix spacecraft assembly clean room. Astrobiology. 2010;10(5):499-508.

42. La Duc MT, Dekas A, Osman S, Moissl C, Newcombe D, Venkateswaran K. Isolation and characterization of bacteria capable of tolerating the extreme conditions of clean room environments. Appl Environ Microbiol. 2007;73(8):2600-11. doi:10.1128/AEM.03007-06.

43. La Duc MT, Nicholson W, Kern R, Venkateswaran K. Microbial characterization of the Mars Odyssey spacecraft and its encapsulation facility. Environ Microbiol. 2003;5(10):977-85.

44. Venkateswaran K, Satomi M, Chung S, Kern R, Koukol R, Basic C, et al. Molecular microbial diversity of a spacecraft assembly facility. Syst Appl Microbiol. 2001;24(2):311-20. http://dx.doi.org/10.1078/0723-2020-00018.

45. La Duc MT, Osman S, Vaishampayan P, Piceno Y, Andersen G, Spry JA, et al. Comprehensive census of bacteria in clean rooms by using DNA microarray and cloning methods. Appl Environ Microbiol. 2009;75(20):6559-67. doi:10.1128/aem.01073-09.

46. Chaudhary N, Marr KA. Impact of Aspergillus fumigatus in allergic airway diseases. Clinical and translational allergy. 2011;1(1):4. doi:10.1186/2045-7022-1-4.

47. Ward MD, Chung YJ, Copeland LB, Doerfler DL. A comparison of the allergic responses induced by Penicillium chrysogenum and house dust mite extracts in a mouse model. Indoor Air. 2010;20(5):380-91. doi:10.1111/j.16000668.2010.00660.x. 
48. Schuster E, Dunn-Coleman N, Frisvad JC, van Dijck PWM. On the safety of Aspergillus niger-a review. Appl Microbiol Biotechnol. 2002;59:426-35.

49. Piñar G, Sterflinger K, Ettenauer J, Quandt A, Pinzari F. A combined approach to assess the microbial contamination of the Archimedes Palimpsest. Microb Ecol. 2015;69:118-34. doi:10.1007/s00248-014-0481-7.

50. Pierson D, Botkin D, Bruce R, Castro V, Smith M, Oubre C, et al. Microbial monitoring of the International Space Station. In: Moldenhauer J, editor. Environmental Monitoring: A Comprehensive Handbook. LLC, River Grove, IL: DHI Publishing; 2012.

51. Chiang YM, Meyer KM, Praseuth M, Baker SE, Bruno KS, Wang CCC. Characterization of a polyketide synthase in Aspergillus niger whose product is a precursor for both dihydroxynaphthalene (DHN) melanin and naphtho-gamma-pyrone. Fungal Genet Biol. 2011;48(4):430-7. doi:10.1016/j.fgb.2010.12.001.

52. Sanchez JF, Somoza AD, Keller NP, Wang CCC. Advances in Aspergillus secondary metabolite research in the post-genomic era. Nat Prod Rep 2012;29(3):351-71. doi:10.1039/c2np00084a.

53. Perry JL. International Space Station bacteria filter element service life evaluation: Marshall Space Flight Center 2005 April 2005 Contract No. NASA/TM-2005-213846.

54. Liu BYH, Rubow KL, McMurry PH, Kotz TJ, Russo D. Airborne particulate matter and spacecraft internal environments. 21st International Conference on Environmental Systems; San Francisco, CAJuly 1991.

55. Katherine T, Steve L, Rachel E. Development of a modified vacuum cleaner for lunar surface systems. 40th International Conference on Environmental Systems. International Conference on Environmental Systems (ICES). Washington DC: American Institute of Aeronautics and Astronautics; 2010.

56. Selbmann L, de Hoog GS, Mazzaglia A, Friedmann El, Onofri S. Fungi at the edge of life, cryptoendolithic black fungi from Antarctic deserts. Stud Mycol. 2005:51:1-32.

57. Yamada S, Ohashi E, Agata N, Venkateswaran K. Cloning and nucleotide sequence analysis of gyrB of Bacillus cereus, B. thuringiensis, B. mycoides, and B. anthracis and their application to the detection of $B$. cereus in rice. Appl Environ Microbiol. 1999;65(4):1483-90.

58. La Duc MT, Satomi M, Agata N, Venkateswaran K. gyrB as a phylogenetic discriminator for members of the Bacillus anthracis-cereus-thuringiensis group. J Microbiol Meth. 2004;56(3):383-94.

59. Kwan K, Cooper M, La Duc MT, Vaishampayan P, Stam C, Benardini JN, et al. Evaluation of procedures for the collection, processing, and analysis of biomolecules from low-biomass surfaces. Appl Environ Microbiol. 2011;77(9):2943-53. doi:10.1128/aem.02978-10.

60. Venkateswaran K, Hattori N, La Duc MT, Kern R. ATP as a biomarker of viable microorganisms in clean-room facilities. J Microbiol Methods. 2003;52(3):367-77.

61. Suzuki MT, Taylor LT, DeLong EF. Quantitative analysis of small-subunit rRNA genes in mixed microbial populations via 5'-nuclease assays. App Environ Microbiol. 2000;66(11):4605-14

62. Dowd SE, Sun Y, Secor PR, Rhoads DD, Wolcott BM, James GA, et al. Survey of bacterial diversity in chronic wounds using pyrosequencing, DGGE, and full ribosome shotgun sequencing. BMC Microbiol. 2008;8:43. doi:10.1186/1471-2180-8-43

63. Caporaso JG, Kuczynski J, Stombaugh J, Bittinger K, Bushman FD, Costello EK, et al. QIIME allows analysis of high-throughput community sequencing data. Nat Methods. 2010;7(5):335-6. doi:10.1038/nmeth.f.303.

64. McDonald D, Price MN, Goodrich J, Nawrocki EP, Desantis TZ, Probst A, et al. An improved Greengenes taxonomy with explicit ranks for ecological and evolutionary analyses of bacteria and archaea. ISME J. 2012;6(3):610-8. doi:10.1038/ismej.2011.139.

65. DeSantis TZ, Hugenholtz P, Larsen N, Rojas M, Brodie EL, Keller K, et al. Greengenes, a chimera-checked 165 rRNA gene database and workbench compatible with ARB. Appl Environ Microbiol. 2006;72(7):5069-72.

66. Edgar RC. Search and clustering orders of magnitude faster than BLAST. Bioinformatics. 2010;26(19):2460-1. doi:10.1093/bioinformatics/btq461.

67. Schloss PD, Gevers D, Westcott SL. Reducing the effects of PCR amplification and sequencing artifacts on 16S rRNA-based studies. PLoS One. 2011;6(12), e27310. doi:10.1371/journal.pone.0027310 PONE-D-11-13353.

68. Bengtsson-Palme J, Ryberg M, Hartmann M, Branco S, Wang Z, Godhe A, et al. Improved software detection and extraction of ITS1 and ITS2 from ribosomal ITS sequences of fungi and other eukaryotes for analysis of environmental sequencing data. Meth Ecol Evol. 2013;4(10):914-9.
69. Edgar RC, Haas BJ, Clemente JC, Quince C, Knight R. UCHIME improves sensitivity and speed of chimera detection. Bioinformatics. 2011;27:2194-200.

70. Nilsson RH, Tedersoo L, Ryberg M, Kristiansson E, Hartmann M, Unterseher $M$ et al. A comprehensive, automatically updated fungal ITS sequence dataset for reference-based chimera control in environmental sequencing efforts. Microbes and Environments (in press). 2015.

71. Nilsson RH, Kristiansson E, Ryberg M, Hallenberg N, Larsson KH. Intraspecific ITS variability in the kingdom fungi as expressed in the international sequence databases and its implications for molecular species identification. Evol Bioinform Online. 2008;4:193-201.

72. Zhang Z, Schwartz S, Wagner L, Miller W. A greedy algorithm for aligning DNA sequences. J Comput Biol. 2000;7(1-2):203-14. doi:10.1089/ 10665270050081478.

73. Benson DA, Clark K, Karsh-Mizrachi I, Lipman DJ, Ostell J, Sayers EW. GenBank. Nucleic Acids Res. 2014;42:D32-7.

74. Abarenkov K, Nilsson RH, Larsson K-H, Alexander IJ, Eberhardt U, Erland S, et al. The UNITE database for molecular identification of fungi-recent updates and future perspectives. New Phytol. 2010;186(2):281-5.

75. Koljalg U, Nilsson RH, Abarenkov K, Tedersoo L, Taylor AF, Bahram M, et al. Towards a unified paradigm for sequence-based identification of fungi. Mol Ecol. 2013;22(21):5271-7. doi:10.1111/mec.12481.

76. Nilsson RH, Tedersoo L, Abarenkov K, Ryberg M, Kristiansson E, Hartmann M, et al. Five simple guidelines for establishing basic authenticity and reliability of newly generated fungal ITS sequences. MycoKeys. 2012;4:37-63.

77. Kuczynski J, Stombaugh J, Walters WA, Gonzalez A, Caporaso JG, Knight R. Using QIIME to analyze 16S rRNA gene sequences from microbial communities. Curr Protoc Bioinformatics. 2011; Chapter 10:Unit 107. doi:10.1002/0471250953.bi1007s36.

78. Altschul SF, Madden TL, Schaffer AA, Zhang J, Zhang Z, Miller W, et al. Gapped BLAST and PSI-BLAST: a new generation of protein database search programs. Nucleic Acids Res. 1997;25(17):3389-402.

79. Anders $\mathrm{S}$, Huber W. Differential expression analysis for sequence count data. Genome Biol. 2010;11(10). doi:10.1186/gb-2010-11-10-r106.

80. Benjamini $Y$, Hochberg $Y$. Controlling the false discovery rate: a practical and powerful approach to multiple testing. J Roy Stat Soc B. 1995;57(1):289-300

81. R-project. R Development Core Team R: A language and environment for statistical computing (http://www.r-project.org/). R Foundation for Statistical Computing, Vienna, Austria. 2011. http://www.r-project.org/.

\section{Submit your next manuscript to BioMed Central and take full advantage of:}

- Convenient online submission

- Thorough peer review

- No space constraints or color figure charges

- Immediate publication on acceptance

- Inclusion in PubMed, CAS, Scopus and Google Scholar

- Research which is freely available for redistribution 